\title{
Novel extra-thoracic VATS minimally invasive technique for management of multiple rib fractures
}

\author{
Natalie N. Merchant ${ }^{1}$, Osita Onugha ${ }^{2}$ \\ ${ }^{1}$ David Geffen School of Medicine, University of California, Los Angeles, Los Angeles, CA, USA; ${ }^{2}$ Department of Thoracic Surgery, John Wayne \\ Cancer Institute, Santa Monica, CA, USA \\ Correspondence to: Osita Onugha, MD, MBA. Department of Thoracic Surgery, John Wayne Cancer Institute, 2121 Santa Monica Blvd. \\ Cardiothoracic Outpatient Clinic, Santa Monica, CA, USA. Email: osita.onugha@providence.org.
}

\begin{abstract}
We report an elderly patient with chronic obstructive pulmonary disease (COPD), Alzheimer's disease and early dementia who presented with multiple displaced rib fractures of left ribs 4 through 9 with flail segments of ribs 4 through 8 and an associated traumatic pneumatocele from rib puncture of the left upper lobe. The decision to treat this patient operatively was based on the presence of flail chest, the patient's age, baseline co-morbidities and limited physiological reserve. Surgical rib fixation is traditionally performed with a thoracotomy incision and open exposure for extra-thoracic rib fixation, however, this patient underwent chest wall stabilization using an extra-thoracic video-assisted thoracic surgery (VATS) technique. We discuss our operative technique using universal rib plating system, optimal retraction for exposure and use of balloon dilation to create an accessible extra-thoracic working space. This surgical approach provided a faster recovery to this patient's baseline with minimal use of narcotics thereby highlighting the impact and importance of this surgical technique for patients presenting with multiple fib fractures, especially the elderly. We show that VATS assisted minimally invasive technique for operative management of multiple rib fractures is a viable surgical option demonstrated by this patient's recovery and return to function with minimal need for pain control despite her advanced age and baseline co-morbidities.
\end{abstract}

Keywords: Flail chest; rib fixation; video-assisted thoracic surgery (VATS); geriatric surgery

Received: 12 April 2018; Accepted: 24 April 2018; Published: 14 May 2018.

doi: 10.21037 /jovs.2018.05.08

View this article at: http://dx.doi.org/10.21037/jovs.2018.05.08

\section{Introduction}

Several studies have demonstrated that operative management of multiple rib fractures can decrease morbidity and mortality and decrease the need for long-term pain control, especially in patients over 65 years of age (1).

\section{Case presentation}

79-year-old female with past medical history of longstanding COPD, Alzheimer's disease, and early stage dementia presented to the emergency department after suffering two consecutive ground level falls. Clinical examination revealed tachypnea, pain at left chest wall and paradoxical chest wall motion. Chest computed tomography (CT) revealed a left pneumothorax, multiple displaced fractures of left ribs 4 through 9 and associated traumatic pneumatocele (Figure 1). A chest tube was placed to evacuate the pneumothorax and the patient was initially managed with pulmonary toilet and a combination of epidural and oral pain medication, but operative management was ultimately decided to be the most beneficial. Threedimensional CT reconstruction is obtained for patients with rib fractures in order to further evaluate the anatomy. This imaging demonstrates the position of the displaced and non-displaced rib fractures prior to surgery (Figure 2).

The patient underwent minimally invasive left chest wall stabilization using extra-thoracic VATS technique. Patient was placed in right lateral decubitus position and double lumen was placed. Considering the subsequent rib fractures involved, a camera was placed to examine the intra-thoracic 

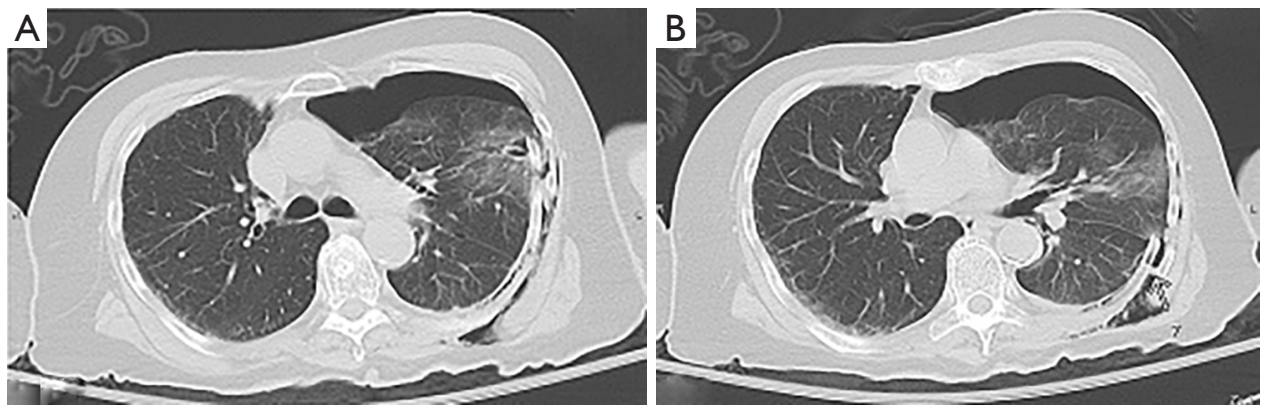

Figure 1 Chest computed tomography. (A) Image showing $5^{\text {th }}$ rib perforation of the left upper lobe causing contusion and traumatic pneumatocele with resulting pneumothorax and subcutaneous emphysema; (B) image showing 7 th rib displaced and puncturing the pleura.
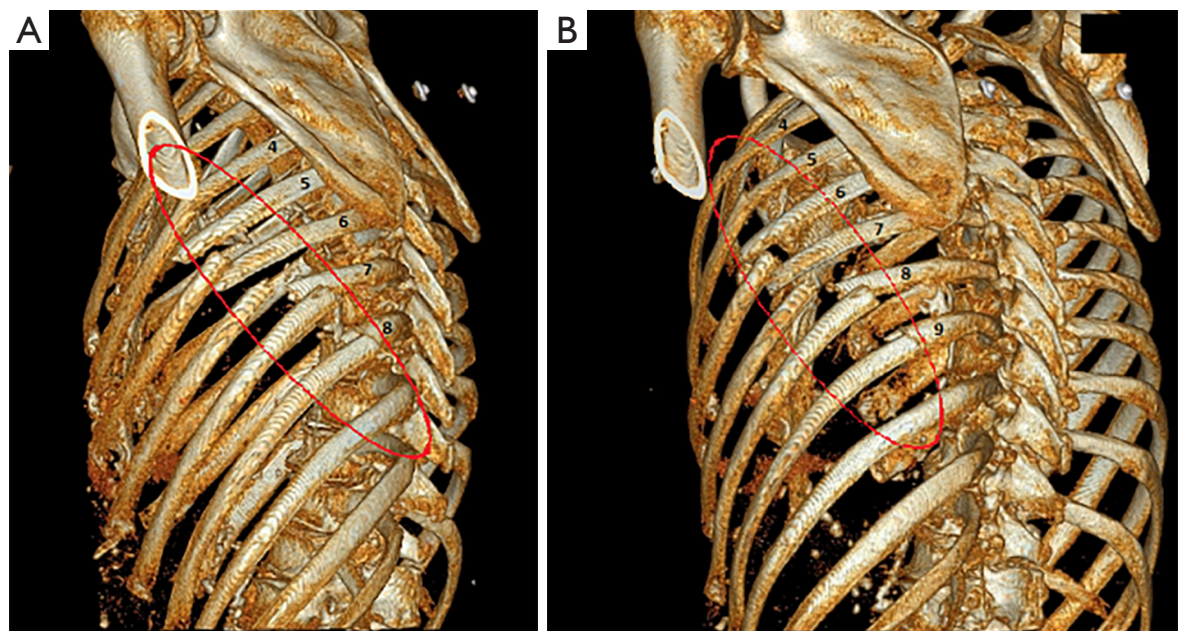

Figure 2 Three-dimensional chest CT reconstruction. (A) Image showing lateral view of non-displaced rib fractures 4 and 8; (B) image showing posterior-lateral view showing non-displaced fractures on rib 4-9, with displaced fractures of ribs 6-8.
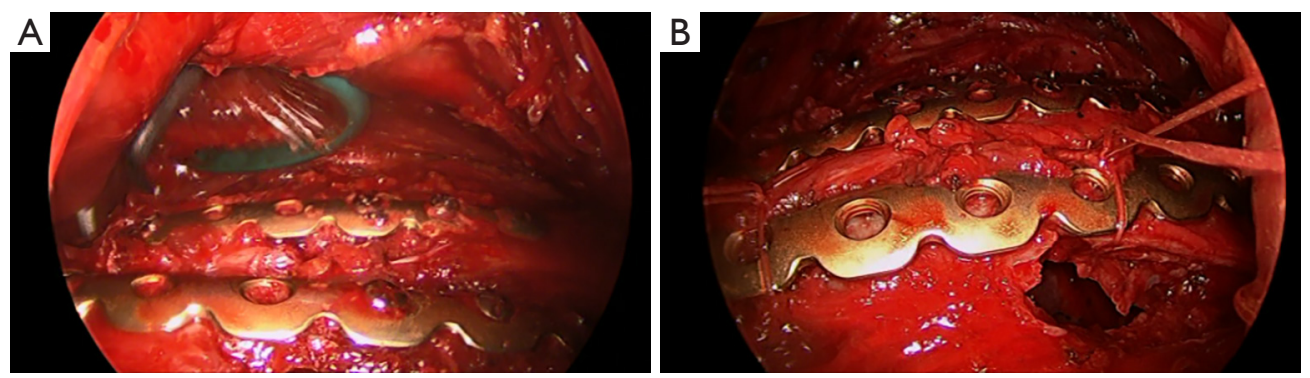

Figure 3 Intra-operative image. (A) Showing the creation of an extra-thoracic space using balloon dilation; (B) showing placement and securing of plates on rib 5 . Rib 4 plating is complete.

space. The chest wall was palpated to determine chest wall instability and optimal incision placement. An extra-thoracic space was created between the chest wall musculature and the superficial aspect of the ribs using a space maker balloon and wound protector (Figure 3A). Wound retractors were used to maintain the extra-thoracic space. After the rib fractures were identified, fixation of ribs $4-8$ began in a cranial-tocaudal fashion. Positioning of the titanium plate/screw 


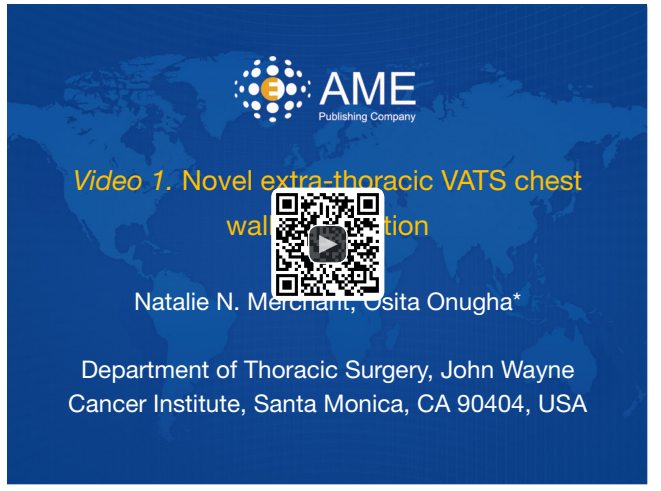

Figure 4 Novel extra-thoracic VATS chest wall stabilization (2). Available online: http://www.asvide.com/article/view/24744

system was secured by placing sutures bilaterally around the flail segments (Figure 3B). Placement of the screws and accessibility of the screw holes was achieved by a combination of right-angle and straight-angle electric drills and was easily accessible through the exposure (Figure 4). Once good operative fixation was obtained and the patient was extubated in the operating room. The chest tube was removed on postoperative day (POD) 2 and JP drain on POD3. The patient was ready for discharge on POD 3 with chest radiograph demonstrating stable fixation and her post-operative pain was well controlled on acetaminophen and ibuprofen (Figure 5).

\section{Discussion}

Rib fractures are common injuries and occur in up to $39 \%$ of patients with blunt chest trauma. These fractures are routinely treated non-operatively, however this management strategy has higher rates of long-term morbidity, especially in elderly patients $(3,4)$. These findings may be due to increase in medical co-morbidities at baseline in addition to limited physiologic reserve, making elderly patients a particularly vulnerable population. It is clear how the patient, with consecutive fractures in ribs 4-9, would benefit from operative fixation (5).

Adequate pain management is essential for operative and non-operative management of multiple rib fractures as patients need chest wall expansion and production of cough to clear secretions to decrease incidence of pneumonia. Shelat and colleagues demonstrate that after non-operative management of rib fractures and 8 weeks of outpatient analgesia, $26 \%$ of patients are not adequately treated and require long-term use of analgesics while our patient
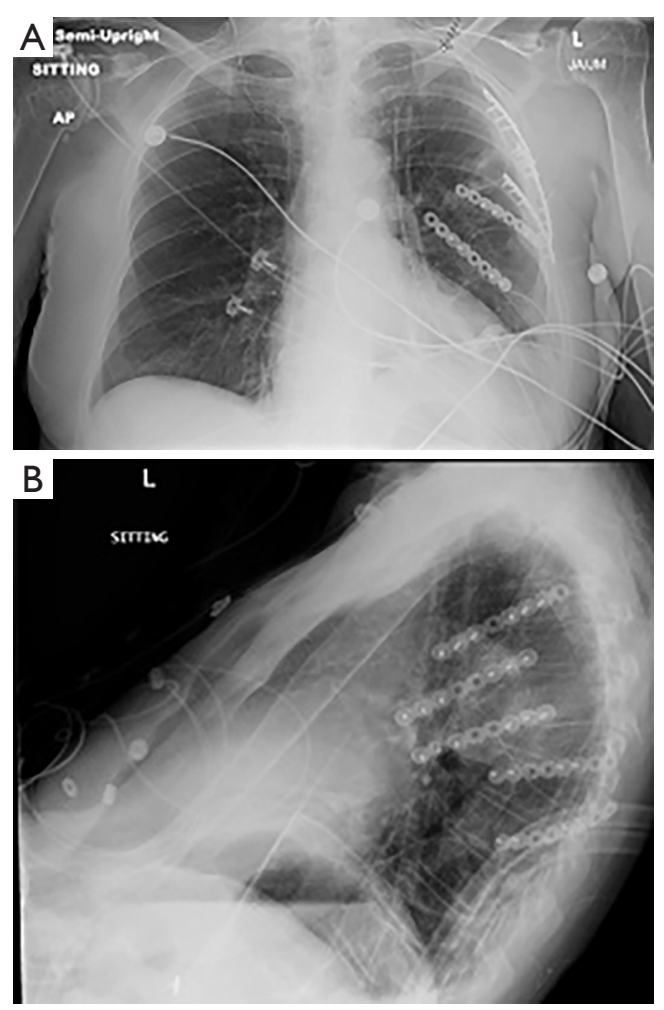

Figure 5 Post-operative chest radiograph. (A) In anterior-posterior view showing stable rib fixation of ribs 4-8; (B) in lateral view showing stable rib fixation of ribs 4-8.

returned to her baseline function at her 1-week postoperative outpatient clinic visit (6).

Although there have been two articles published regarding the use of VATS to perform rib fixation surgery, this is the first publication of surgical rib fixation using extra-thoracic VATS incisions for placement of rib plates for multiple subsequent rib fractures and flail chest. Typically, surgical rib fixation is performed with a thoracotomy incision, but this VATS-assisted minimally invasive surgical approach provided a faster recovery to this patient's baseline with minimal post-operative neuralgia or soft tissue damage.

\section{Conclusions}

We show that VATS assisted minimally invasive technique for operative management of multiple rib fractures is a viable surgical option demonstrated by this patient's recovery and return to function with minimal need for pain control despite her advanced age and baseline co-morbidities. 


\section{Acknowledgements}

None.

\section{Footnote}

Conflicts of Interest: The authors have no conflicts of interest to declare.

Informed Consent: Written informed consent was obtained from the patient for publication of this Case Report and ant accompanying images. A copy of the written consent is available for review by the Editor-in-Chief of this journal.

\section{References}

1. Bulger EM, Arneson MA, Mock CN, et al. Rib fractures in

doi: $10.21037 /$ jovs.2018.05.08

Cite this article as: Merchant NN, Onugha O. Novel extrathoracic VATS Minimally invasive technique for management of multiple rib fractures. J Vis Surg 2018;4:103. the elderly. J Trauma 2000;48:1040-6.

2. Merchant NN, Onugha O. Novel extra-thoracic VATS chest wall stabilization. Asvide 2018;5:513. Available online: http://www.asvide.com/article/view/24744

3. Dehghan N, De Mestral C, Mckee MD, et al. Flail chest injuries: a review of outcomes and treatment practices from the National Trauma Data Bank. J Trauma Acute Care Surg 2014;76:462-8.

4. Fabricant L, Ham B, Mullins R, et al. Prolonged pain and disability are common after rib fractures. Am J Surg 2013;205:511-5.

5. Pieracci FM, Rodil M, Stovall RT, et al. Surgical stabilization of severe rib fractures. J Trauma Acute Care Surg 2015;78:883-7.

6. Shelat VG, Eileen S, John L, et al. Chronic pain and its impact on quality of life following a traumatic rib fracture. Eur J Trauma Emerg Surg 2012;38:451-5. 